\title{
Anti-inflammatory Effects of Naïve Stem Cells Dampen Systemic/ Compartmental Overreactive Immune Responses
}

\author{
Wolters ECh' ${ }^{1}$, de Hoo K' ${ }^{2}$, Kramer BW ${ }^{3}$, de Munter JPJM ${ }^{2,4}$ \\ 'Department of Neurology, UniversitatsSpital Zurich, Zurich, Switzerland \\ ${ }^{2}$ Neuroplast BV, Urmond, The Netherlands \\ ${ }^{3}$ Department of Paediatrics, Maastricht University Medical Centre, Maastricht, The Netherlands \\ ${ }^{4}$ School for Mental Health and NeuroScience, Maastricht University, Maastricht, The Netherlands
}

Article Info

\section{Article Notes}

Received: January 11, 2021

Accepted: March 30, 2021

\section{*Correspondence:}

Dr. Johannes P.J.M. de Munter, School for Mental Health and NeuroScience, Maastricht University, Maastricht, The Netherlands; Email: h.demunter@neuroplast.com.

(c) 2021 de Munter JPJM. This article is distributed under the terms of the Creative Commons Attribution 4.0 International License.

\section{Keywords}

Systemic cytokine release syndrome

Compartmental cytokine release syndrome

SARS-CoV-19

Traumatic spinal cord Injury

Bone marrow-derived naïve stem cells

\section{ABSTRACT}

A cytokine release syndrome (CRS), associated with elevated circulating levels of several cytokines including interleukin (IL)- 6 and interferon (IFN)- $v$, might be seen in some infectious insults, for instance in severe acute respiratory syndrome (SARS) induced by Coronavirus (Cov)-2, as well as following administration of natural and bispecific antibodies and, more recently, following adoptive T-cell therapies for cancer. Normally, inflammatory conditions activate the innate and adaptive immune systems, which results in the release of cytokines, responsible for the phagocytosis of apoptotic vesicles and resolution of inflammation. Pro-inflammatory cytokines such as IL-1 $\beta$, tumor necrosis factor alpha (TNF $\alpha$ ) and, especially in chronic inflammatory diseases, autoimmune diseases, cancer and cytokine storms, IL-6 play crucial roles in inflammation. In some instances, however, this release gets out of hand, and features of overzealous immune responses (macrophage activation syndromes) might occur, leading to cytokine release syndromes (CRS) with inflammatory signs such as fever, fatigue, nausea, and sometimes secondary organ dysfunction or multi-organ failure. Apart from specific vaccines and maybe the anti-viral remdesivir and/or dexamethasone for treatment of CRS, there are no convincing disease-modifying interventions. So far, though, nonantiviral and immune-targeted interventions, also affecting non-target cells, were found associated with many side effects. A more targeted or focused approach is thus needed. Pending the site of the CRS-inducing insult, CRSs may occur systemic or compartmental. Recently, preclinical research yielded a beneficial anti-inflammatory effect of fresh naive bone marrow-derived stem cells (bm-SCs) in the treatment of various compartmental CRSs in the immuneprivileged central nervous system (CNS). Therefore, it is argued that bm-SCs might also play a disease-modifying role in the systemic CRS. Bm-SCs have the advantage of targeting only the cells of interest as they are very selective in their actions. In addition, they actively move to the sight of inflammation.

\section{Over Reactive Immune Responses}

In some inflammatory conditions a disbalance between pro- and anti-inflammatory cytokines (CRS) might result in a cytokine release syndrome (CRS). Those conditions include both infectious (sepsis, viremia, herpes, Ebola, malaria, Dengue, Lassa and SARS-Cov-19) and sterile insults (monogenic and auto-immune disorders, organ transplantation, immunotherapies like monoclonal antibodies or chimere antigen receptor (CAR)-T cells for cancer, burns, ischemia, and trauma $)^{1-3}$. When the immune system is fighting (sterile) inflammation, chemokines signal immune cells such as T-cells and macrophages, to travel to the site of inflammation, and to stimulate/enhance local production of cytokines. In corona virusinduced severe acute respiratory syndrome (SARS) or Middle East 
respiratory syndrome (MERS), for instance, viruses bind to alveolar epithelial cells, and then activate the innate and adaptive immune systems, which results in the release of many cytokines, including (IFN) $-\gamma$ and IL- $6^{1}$. Normally, this process is adequately controlled by the anti-inflammatory mechanisms of the body. However, in some instances, this mechanism gets out of hand, and features of overzealous immune responses (CRS) might occur. This phenomenon might explain why some people have a severe reaction to for instance coronaviruses while others only experience mild symptoms. The reason why younger people are less involved, though, is still an enigma. CRS may be best formulated as an infectious or sterile-induced production of circulating cytokines beyond a normal response, to a specific condition, leading to inflammatory signs with fever, severe fatigue, nausea and in some cases even secondary organ dysfunction or multi-organ failure ${ }^{4}$.

The precise reason for this excessive inflammatory response is not entirely understood but may be caused by new or specific highly pathogenic conditions, able to initiate a macrophage activation syndrome (MAS). Cytokines play an important role in normal immune responses but when they overshoot their target, due to a MAS, they can be extremely harmful. In those conditions, the expression of a healthy immune system results in the pathological release of multiple inflammatory mediators (cytokines, oxygen free radicals, and coagulation factors). These include both pro-inflammatory mediators such as TNF (an inflammatory cytokine produced by macrophages/ monocytes during acute inflammation, responsible for a diverse range of signaling events within cells, leading to necrosis or apoptosis), IL-1 (a group of 11 cytokines that plays a central role in the regulation of immune and inflammatory responses to infections or sterile insults, stimulating phagocytosis and programmed cell death) and IL-6 (a pleiotropic cytokine, secreted by $\mathrm{T}$ cells and macrophages o activate immune responses during infections or after trauma), as well as anti-inflammatory mediators such as IL-1 receptor antagonists and the cytokine synthesis inhibitory factor IL-10 (a cytokine with potent anti-inflammatory properties that plays a central role in limiting host immune response to pathogens, thereby preventing damage to the host and maintaining normal tissue homeostasis). So, it is not the illiciting pathogenic insult itself but rather the overreactive immune response to this condition, which induces a cytokine release syndrome.

A CRS can be systemic or compartmental, such as in immune-privileged areas such as the eyes, joints and the central nervous system. Following traumatic brain injury (TBI) and spinal cord injury (SCI), there are no diseasemodifying interventions, but preclinical research suggests a beneficial (anti-inflammatory) effect of autologous naive stem cells to treat this overactive inflammatory condition ${ }^{5}$.

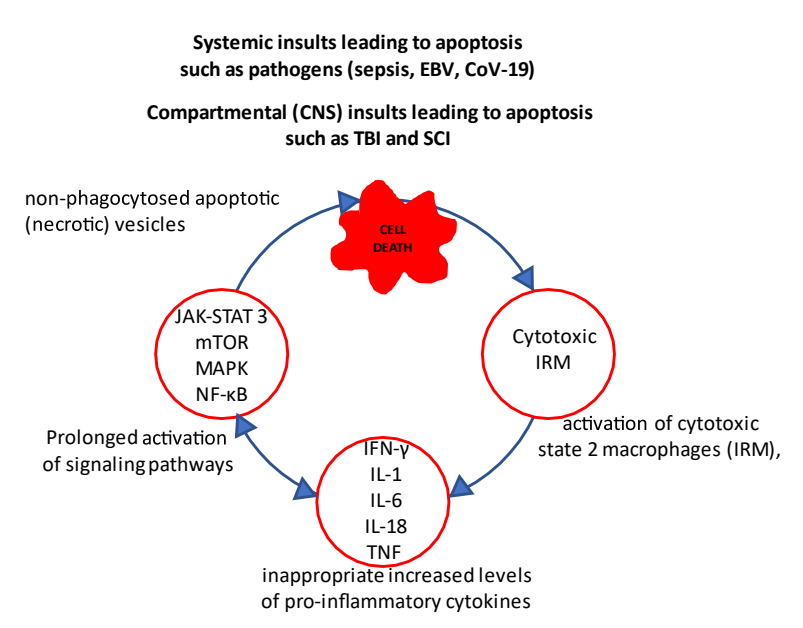

Figure 1: Schematic overview of a cytokine release syndrome: Schematic drawing of systemic and compartmental (immuneprivileged compartments such as eyes, joints and the central nervous system) cytokine release syndromes. These syndromes involve inappropriate immune cell hyperactivation (in systemic insults mainly due to a relatively high content of interferonresponsive macrophages (IRM), and in compartmental CNS insults mainly of interferon-responsive microglia (IRM) and less macrophages, which enter the compartment through the leaking blood-brain-barrier) with elevated levels of proinflammatory cytokines, mainly interferon (IFN)- $\gamma$, interleukins (IL) 1, 6 and 18, and tumor necrosis factor (TNF), inducing prolonged activation of signaling pathways JAK-STAT (Janus kinase-signal transducer and activator of transcription) 3, MAPK (mitogen-activated protein kinase), mTOR (rapamycin), and NF-KB (nuclear factor kappa-light-chain-enhancer of activated $B$ cells).

Stem cells are not only acting on local concentrations of circulating pro- and anti-inflammatory proteins, they are rather selective in their actions, thus sparing non-target tissue cells ${ }^{6}$. The risk of stem cells for eventual adverse events in humans can be comprehensively reduced when applying fresh non-substantial manipulated autologous stem cells ${ }^{7}$.

\section{Compartmental Overreactive Immune Responses (TBI, SCI)}

Pro-inflammatory cytokines play an essential role in maintenance of normal and repair functions, for instance in the central nervous system compartment, after TBI and SCI. Massive and uncontrolled release of these cytokines such as IL- $1 \beta$ and TNF- $\alpha$, and especially IL-6, though, may result in a great deal of collateral damage around the site of the injury ${ }^{8}$. After an initial acute injury-induced necrosis, a profound and uncontrolled, local secondary inflammatory reaction will develop, characterized by the abundant release of several cytokines, mainly IL-6, with pro- and anti-inflammatory functions ${ }^{9-12}$. In terms of molecular pathogenesis, traumatic injuries of brain and spinal cord share a negative contribution of non-neuronal 
cells (immune cells, glial cells) expressing and activating the inflammatory process as a significant commonality ${ }^{13,14}$. Microglia, the resident immune cells in the CNS, comprise an entire spectrum of phenotypes that span the range from deleterious to regulatory to remodeling effects. Gene expression analyses led to the identification of homeostatic state 1 microglia and environmental-dependent, disease associated, reactive state 2 and state 3 microglia. State 2 microglia are the classical acute necrosis-activated cytotoxic microglia (interferon response microglia: IRMs), affecting neuronal survival and secreting pro-inflammatory TNF, IL- $1 \beta$ and IL-12, and nitric oxide. State 3 microglia are the alternatively activated, cytoprotective microglia (activated response microglia: ARM) that express genes involved in innate immune response, thus supporting an antiinflammatory response, and preventing classical microglial activation. ARMs secrete the anti-inflammatory cytokines IL-4 (stimulating activated B-cell and T-cell proliferation, and the differentiation of $\mathrm{B}$ cells into plasma cells), the immunoregulatory IL-13 (regulates the function of human $B$ cells and monocytes), and TGF- $\beta$. Once the inciting inflammatory event has been adequately resolved, a lower production of pro-inflammatory cytokines will convert IRMs into ARMs which facilitate phagocytosis of cell debris, promote tissue repair, and support cell survival ${ }^{15}$. However, when the pathogenic stimulus for some reason cannot be adequately cleared, a persistent IRM response will follow ${ }^{16}$. As a consequence, a progressive unintended injury might follow, as is the case in many neurodegenerative disorders. This condition is visualized in the perpetual cell deathinducing process in figure 1.

In the central nervous system, pending the function of the blood-brain-barrier, CRS may lead to highly elevated levels of IL-6 (1.400-355.000 pg/ml), normally marginally detectable $(10-230 \mathrm{pg} \mathrm{ml})$ as compared to corresponding serum levels $(0-11.000 \mathrm{pg} / \mathrm{ml})^{9,17-19}$. Significant higher levels were also seen for other cytokines, including IL- $1 \alpha$, IL-1 $\beta$, IL-8, IL-10, and IL-12 ${ }^{18,20,21}$. Some of these cytokines can have both beneficial as well as harmful effects on the brain. The timing of the rise in cytokines may be crucial.

Acute or chronic pathological cytokine release is established in some neurologic conditions, spinal/cerebral trauma, amyotrophic lateral sclerosis (ALS), Alzheimer's and Parkinson's disease as well as multiple sclerosis, and explained as an underlying cause of the non-infectious pathology ${ }^{22-24}$. Here, the body is not capable to adequately handle these inflammatory processes, which then become chronic, and progressively affect neurological functioning. Ultimately, in animal models for amyotrophic lateral sclerosis (ALS) and spinal cord injury (SCI) this process could be aborted, and pro-inflammatory cytokine levels lowered by an intervention with $\mathrm{Bm}-\mathrm{SCs}^{12,25}$. In spinal cord injury, acute injury-induced apoptosis is followed by a necrosis-induced secondary inflammatory response, deteriorating the direct trauma-induced neurological functioning ${ }^{11}$. Activated microglia and macrophages play an important role in this secondary inflammation. Cytotoxic IRMs (see figure 1), here were found responsible for the enhanced production of pro-inflammatory cytokines ${ }^{5}$. The intrathecal application of a standardized human Bm-SC product (fresh not-manipulated autologous bone marrow containing high concentrations of viable hematopoietic and mesenchymal stem cells obtained by centrifugation and positive depletion of erythrocytes, monocytes and lymphocytes, suspended in Ringers lactate, and reduced in volume for intrathecal application: Neuro-Cells, manufactured by Neuroplast BV, The Netherlands) did reduce the levels of pro-inflammatory cytokines as well as the neuropathologic signs in the central nervous system. Most importantly, the resolution of the inflammatory process did improve functional outcome (which was not the case in steroid treated control groups ${ }^{26}$. Indeed, the benefit of Bm-SCs over anti-inflammatory drugs, including steroids, was shown in both spinal cord injury and ALSlike animals ${ }^{25,26}$. The exact mechanisms are still unknown, but the fact that stem cells are not only decision-making cells, moving to and acting upon local concentrations of circulating pro- and anti-inflammatory proteins, is a likely explanation for the beneficial roles of bm-SCs ${ }^{6}$. The risk for eventual adverse events of stem cells, when limited to the exclusive use of non-substantial manipulated autologous stem cells, is negligible?

Another compartment are the synovial spaces of joints in which an overwhelming immune reaction can exacerbate an ongoing arthritis. The onset is still not clear, but in the affected joints, cytokines such as TNF and IL-1 here play an important role in apoptosis and loss of cartilage cells ${ }^{27,28}$. The levels of TNF and IL-1 are increased in patients with rheumatoid arthritis in serum as well as in the synovial fluid.

\section{Systemic Overreactive Immune Responses (SARS CoV-2)}

Lately we have been faced by the devastating effects of COVID-19 infections in which fatalities were linked to a dysregulated host immune response to a severe acute respiratory syndrome (SARS-CoV-2). In the most serious affected patients, SARS-induced MAS displayed significantly increased plasma levels of several cytokines, chemokines, and inflammatory markers (such as C-reactive protein, procalcitonin, and ferritin), levels correlating with serious systemic signs of inflammation, and sometimes organ dysfunction or multi-organ failure with fatal outcome ${ }^{29-33}$. Severe COVID-19 and SARS infections are notable for severe T-cell depletion. In COVID-19, there is unique suppression of interferon signaling by infected respiratory tract cells with intact cytokine signaling. A decreased naïve T-cell 
response likely contributes to an excessive inflammatory response and increases the odds of a cytokine storm ${ }^{8}$. Serum cytokine levels that were found elevated in patients with CoV-19-associated hyperreactive immune responses included IL-1 $\beta$, IL-6, TNF, IFN- $\gamma$ and IFN- $\gamma$-induced IP-10, macrophage inflammatory protein (MIP) $1 \alpha$ and $1 \beta$, and vascular endothelial growth factor (VEGF) ${ }^{34,35}$. Especially IL-6 is hypothesized as one of the main mediators ${ }^{1,32,36,37}$. The pooled mean serum interleukin- 6 concentration in these patients was about 100 times higher $(3110.5 \mathrm{pg} /$ $\mathrm{mL}$ ) when compared to Covid patients not suffering this overreactive immune reaction $(36.7 \mathrm{pg} / \mathrm{mL})^{38}$.

In addition to the activated immune cells and elevated systemic cytokine levels, other clinical and laboratory abnormalities were found to predict worsening outcomes in CoV-19, such as an elevated C-reactive protein, hypoalbuminemia, and renal dysfunction ${ }^{38-40}$. Using a logistic regression model to analyze the factors influencing the severity of the disease, Wang and colleagues identified 3 of 19 predictive variables as significant (white blood cells, IL-10, and IL-6 levels) using the new Chinese National Health Commission Coronavirus Pneumonia Classification, and 2 of 19 predictive variables as significant (lower CD3 percentages and higher IL-6 levels) using the Sequential Organ Failure Assessment (SOFA) score. The multivariable regression analysis results showed only IL-6 levels as indicators related to the severity of Covid patients in both classifications $^{32}$.

\section{Treatment of Overreactive Immune Responses}

\section{Systemic cytokine release syndromes}

Pending the severity of cytokine release syndromes, supportive treatment may be necessary to protect organs and prevent further damage. Symptomatic treatment includes intravenous fluids, oxygen or ventilator support, medicines to support heart function, blood product transfusions, medicine or dialysis for the kidneys. Cantini et al. studied the reports of antiviral therapies in Cov19 patients, and concluded that only Remdesevir, not Lopinavir/ritonavir, Favipiravir, Atazanavir, Interferon $\alpha 2 b$, Ribavirin or Umifenovir reduced both some time to recovery and mortality ${ }^{41}$. IL- 6 expression, activated by IL$1 \beta$ and TNF $\alpha$, plays an important role in CRS. Although anti-IL- 6 antibodies should lower IL- 6 expression and thus dampen the downstream IL-6 signaling pathways ${ }^{1.42}$, as in the treatment of rheumatoid arthritis, Castleman's disease and CAR-T-induced CRS ${ }^{1}$, IL-6R blockers Tocilizumab, Sarilumab, and Siltuximab have been ineffective $e^{41,43-48}$. Cantini et al. also evaluated the results of other non-antiviral and immune targeted agents. They concluded that both anakinra and dexamethasone reduced mortality, that results from chloroquine and hydroxychloroquine use were inconclusive; and that Baricitinib resulted in a significant reduction of intensive care unit admission and deaths ${ }^{41}$. The promising results of anakinra, though, awaits confirmation, and corticosteroids, which reduce inflammation without targeting a specific cytokine, as in the treatment of TBI and SCIs were found to increase mortality risk and to delay recovery with a longer hospital stay ${ }^{49,50}$.

Although IL-6 blockade does not work very well in viral-induced CRS, the treatment of CRS in response to immunotherapies like monoclonal antibodies or CAR-T cells for cancer is very well alleviated by anti-IL-6 interventions. In short: symptomatic treatments, maybe antiviral interventions with remdesivir excluded, the treatment of overreactive immune responses is still enigmatic.

\section{Compartmental cytokine release syndromes}

In the central nervous system, microglia, the resident immune cells in the CNS, comprise an entire spectrum of environment-adapted phenotypes that span the range from deleterious to regulatory to remodeling effects. Although microglial activation provides a first defense against acute injury or infection, in these cases chronic or excessive activation is considered highly detrimental ${ }^{16}$. Indeed, in TBI and SCI, direct trauma-induced necrosis is followed by a secondary inflammatory reaction, which incites an excessive IRM response that will eliminate even the surviving cells in the immediate vicinity (the penumbra). In brain and spinal cord injuries, unfortunately, treatment with anti-inflammatory drugs have produced mixed results at best and can produce serious adverse events, unless the result of insufficient dosage or bioavailability, delayed time points of delivery, and/or or the taking-over of the eventually inhibited pathway by other single or downstream pathways ${ }^{5,9,10,51-53}$. As the blood-brain-barrier is usually very tight, the systemic access of these drugs to the brain might also be hampered.

A more recent approach of treating such disorders with intrathecal transplants of fresh bone marrow-derived stromal cells (bm-SCs) seemed more promising ${ }^{12,54,55}$. Intrathecal application of naïve bm-SCs in SCI-rats within $24 \mathrm{~h}$ after the lesioning inhibited the conversion of state-2 (IRM) into state-3 (ARM) microglia, decreased neuronal apoptosis as well as the activators of IL-6 expression (TNF and IL-1 $\beta$ ) in serum and spinal tissue when compared to the vehicle-treated animals $\mathrm{s}^{5,12,55}$. These bm-SCs were found to dampen the downstream IL-6 signaling pathways, with improved clinical outcomes in experimental SCI-rats with evident features of a cytokine-driven hyperimmune reaction.

In case of another compartmental CRS, rheumatoid arthritis, one of the interventional targets is to lower TNF concentration by specific TNF- $\alpha$ inhibitors such as infliximab or certolizumab ${ }^{56,57}$. Despite this specific approach, there is still a non-responder rate of more than 
Systemic apoptosis

IV
Compartmental (CNS) apoptosis

ITh

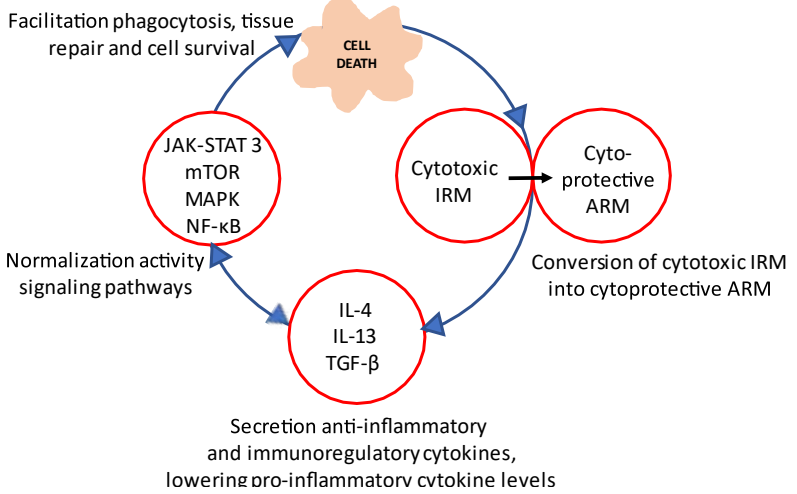

Figure 2: Schematic overview of the effect of bm-SCs in cytokine release syndromes: Schematic drawing of the effect of an intravenous (IV) or intrathecal (ITh) intervention with naive bm-derived stem cells in systemic respectively compartmental cytokine release syndromes (CRS). Stem cells promote the polarization of state-2 interferon-responsive microglia (IRM) into state- 3 activated response microglia (ARM) macrophages, which secrete anti-inflammatory and immunoregulatory cytokines (IL-4, IL-13 and tumor growth factor TGF- $\beta$ ) and normalize the activity of the signaling pathways. Thus, stem cells resolve the inciting inflammatory event, and will facilitate phagocytosis of cell debris, promoting tissue repair, and supporting cell survival.

$30 \%{ }^{58}$, which make this kind of therapy less effective. The experience with stem cells in rheumatoid arthritis is limited but, in general, their (immune-modulating) effects have led to a considerable improvement in the management of RA patients ${ }^{59}$.

\section{Conclusion}

Cytokine release/secretion syndromes can be the endpoint of many different triggers ranging from immunotherapies to infectious diseases. It is not surprising that there is, yet no general therapy established, since the causes are so numerous and different in kind. Preclinical research suggests a beneficial (anti-inflammatory) effect of naive bm-derived stem cells in the treatment of this overactive inflammatory condition in the central nervous system compartment ${ }^{5}$. However, it is even in well controlled experimental models not clear, whether these anti-inflammatory effects are mediated by the resident cells if the stem cells have quickly passed their effects onto those cells. The inherent benefit of bm-derived stem cells over a drug modulating a single inflammatory effector pathway has been shown in head-to-head comparisons. An alternative explanation could be the activation of pathways higher up in the inflammatory cascades. Bm-SCs are decision-making cells that coordinate their operations with their immediate environment $t^{6,60}$. Their cell-to-cell communication here may play a major role and future research must elucidate the content of stem cell-secreted EVs in modulating inflammatory micro-environment(s) and the systemic effect thereof. If the effects of bm-SCs are directly related to the way of administration and the differentiation state of the stem cells, naïve bm-derived stem cells may be superior ${ }^{5}$. Therefore, subsequent studies on the systemic intravenous application of bm-derived stem cells in systemic CRS, and in compartmental CRS diseases via a direct injection (intercompartmental like intrathecal or injections in the joint) need to be done to address this question.

In acute SCI animal models, the intrathecal application of bm-SCs was found to reduce the initial increase of IL-1 $\beta$, TNF and IL- 6 levels, and thus to prevent for the secondary inflammation-induced neurological deficits. This effect might be exerted by the stem cells immediately after application or, indirectly, after passing their effects to resident cells.

\section{Acknowledgment}

The research is done by Neuroplast bv and Neuroplast bv receives an innovation loan of the Ministry of economic affairs of the Netherlands. No additional funding is involved.

\section{Conflict of Interest Statement}

Johannes de Munter is CEO of Neuroplast bv and has a staff position at the department of Neuroscience and Mental Health at the University Maastricht, The Netherlands. Kimberly de Hoo is employed by Neuroplast bv as clinical associate.

\section{References}

1. Zhang $\mathrm{C}, \mathrm{Wu} \mathrm{Z}$, Li JW, et al. Cytokine release syndrome in severe COVID-19: interleukin-6 receptor antagonist tocilizumab may be the key to reduce mortality. Int J Antimicrob Agents 2020; 55(5): 105954.

2. Chousterman BG, Swirski FK, Weber GF. Cytokine storm and sepsis disease pathogenesis. Semin Immunopathol 2017; 39(5): 517-28.

3. Shimabukuro-Vornhagen A, Godel P, Subklewe M, et al. Cytokine release syndrome. J Immunother Cancer 2018; 6(1): 56.

4. Fajgenbaum DC, June CH. Cytokine Storm. N Engl J Med 2020; 383(23): 2255-73.

5. de Munter J, MeyJ, Strekalova T, et al. Why do anti-inflammatory signals of bone marrow-derived stromal cells improve neurodegenerative conditions where anti-inflammatory drugs fail? J Neural Transm (Vienna) 2020; 127(5): 715-27.

6. Fischbach MA, Bluestone JA, Lim WA. Cell-based therapeutics: the next pillar of medicine. Sci Transl Med 2013; 5(179): 179 ps7.

7. de Munter JP, Wolters EC. Autologous stem cells in neurology: is there a future? J Neural Transm 2013; 120(1): 65-73.

8. Schwartz MD, Emerson SG, Punt J, et al. Decreased Naive T-cell Production Leading to Cytokine Storm as Cause of Increased COVID-19 Severity with Comorbidities. Aging Dis 2020; 11(4): 742-5.

9. Morganti-Kossman MC, Lenzlinger PM, Hans V, et al. Production of cytokines following brain injury: beneficial and deleterious for the damaged tissue. Mol Psychiatry 1997; 2(2): 133-6. 
10. Ziebell JM, Morganti-Kossmann MC. Involvement of pro- and antiinflammatory cytokines and chemokines in the pathophysiology of traumatic brain injury. Neurotherapeutics 2010; 7(1): 22-30.

11. Silva NA, Sousa N, Reis RL, etal. From basics to clinical: a comprehensive review on spinal cord injury. Prog Neurobiol 2014; 114: 25-57.

12. de Munter JP, Beugels J, De Munter S, et al. Standardized human bone marrow-derived stem cells infusion improves survival and recovery in a rat model of spinal cord injury. J Neurol Sci 2019; 402: 16-29.

13. Lewis CA, Manning J, Rossi F, et al. The Neuroinflammatory Response in ALS: The Roles of Microglia and T Cells. Neurol Res Int 2012; 2012: 803701.

14. Orsini M, Oliveira AB, Nascimento OJ, et al. Amyotrophic Lateral Sclerosis: New Perpectives and Update. Neurol Int 2015; 7(2): 5885.

15. Khalid SI, Ampie L, Kelly R, et al. Immune Modulation in the Treatment of Amyotrophic Lateral Sclerosis: A Review of Clinical Trials. Front Neurol 2017; 8: 486

16. Hammond TR, Dufort C, Dissing-Olesen L, et al. Single-Cell RNA Sequencing of Microglia throughout the Mouse Lifespan and in the Injured Brain Reveals Complex Cell-State Changes. Immunity 2019; 50(1): 253-71 e6.

17. Arand M, Melzner H, Kinzl L, et al. Early inflammatory mediator response following isolated traumatic brain injury and other major trauma in humans. Langenbecks Arch Surg 2001; 386(4): 241-8.

18. Kushi H, Saito T, Makino K, et al. IL-8 is a key mediator of neuroinflammation in severe traumatic brain injuries. Acta Neurochir Suppl 2003; 86: 347-50.

19. Winter CD, Pringle AK, Clough GF, et al. Raised parenchymal interleukin-6 levels correlate with improved outcome after traumatic brain injury. Brain 2004; 127(Pt 2): 315-20.

20. Helmy A, Carpenter KL, Menon DK, et al. The cytokine response to human traumatic brain injury: temporal profiles and evidence for cerebral parenchymal production. J Cereb Blood Flow Metab 2011; 31(2): 658-70.

21. Helmy A, De Simoni MG, Guilfoyle MR, et al. Cytokines and innate inflammation in the pathogenesis of human traumatic brain injury. Prog Neurobiol 2011; 95(3): 352-72.

22. Nagatsu T, Mogi M, Ichinose H, et al. Cytokines in Parkinson's disease. J Neural Transm Suppl 2000; (58): 143-51.

23. Navikas V, Link H. Review: cytokines and the pathogenesis of multiple sclerosis. J Neurosci Res 1996; 45(4): 322-33.

24. Swardfager W, Lanctot $\mathrm{K}$, Rothenburg L, et al. A meta-analysis of cytokines in Alzheimer's disease. Biol Psychiatry 2010; 68(10): 93041.

25. de Munter J, Shafarevich I, Liundup A, et al. Neuro-Cells therapy improves motor outcomes and suppresses inflammation during experimental syndrome of amyotrophic lateral sclerosis in mice. CNS Neurosci Ther 2019.

26. Romero-Ramirez L, Wu S, de Munter J, et al. Treatment of rats with spinal cord injury using human bone marrow-derived stromal cells prepared by negative selection.J Biomed Sci 2020; 27(1): 35.

27. Moelants EA, Mortier A, Van Damme J, et al. Regulation of TNF-alpha with a focus on rheumatoid arthritis. Immunol Cell Biol 2013; 91(6): 393-401.

28. Farrugia M, Baron B. The role of TNF-alpha in rheumatoid arthritis: a focus on regulatory T cells. J Clin Transl Res 2016; 2(3): 84-90.

29. Group RC, Horby P, Lim WS, et al. Dexamethasone in Hospitalized Patients with Covid-19 - Preliminary Report. N Engl J Med 2020.

30. McGonagle D, Sharif K, O'Regan A, et al. The Role of Cytokines including Interleukin-6 in COVID-19 induced Pneumonia and Macrophage
Activation Syndrome-Like Disease. Autoimmunity reviews 2020; 19(6): 102537.

31. Moore JB, June CH. Cytokine release syndrome in severe COVID-19. Science 2020; 368(6490): 473-4.

32. Wang C, Fei D, Li X, et al. IL-6 may be a good biomarker for earlier detection of COVID-19 progression. Intensive Care Med 2020; 46(7): 1475-6.

33. Zhou F, Yu T, Du R, et al. Clinical course and risk factors for mortality of adult inpatients with COVID-19 in Wuhan, China: a retrospective cohort study. Lancet 2020; 395(10229): 1054-62.

34. Huang C, Wang Y, Li X, et al. Clinical features of patients infected with 2019 novel coronavirus in Wuhan, China. Lancet 2020; 395(10223): 497-506.

35. Zhu Z, Cai T, Fan L, et al. Clinical value of immune-inflammatory parameters to assess the severity of coronavirus disease 2019. International journal of infectious diseases : IJID : official publication of the International Society for Infectious Diseases 2020; 95: 332-9.

36. Aziz M, Fatima R, Assaly R. Elevated interleukin- 6 and severe COVID-19: A meta-analysis. Journal of medical virology 2020; 92(11): 2283-5.

37. Del Valle DM, Kim-Schulze S, Huang HH, et al. An inflammatory cytokine signature predicts COVID-19 severity and survival. Nat Med 2020; 26(10): 1636-43.

38. Leisman DE, Ronner L, Pinotti R, et al. Cytokine elevation in severe and critical COVID-19: a rapid systematic review, meta-analysis, and comparison with other inflammatory syndromes. Lancet Respir Med 2020; 8(12): 1233-44.

39. Caricchio R, Gallucci M, Dass C, et al. Preliminary predictive criteria for COVID-19 cytokine storm. Ann Rheum Dis 2021; 80(1): 88-95.

40. Mathew D, Giles JR, Baxter AE, et al. Deep immune profiling of COVID-19 patients reveals distinct immunotypes with therapeutic implications. Science 2020; 369(6508).

41. Cantini F, Goletti D, Petrone L, et al. Immune Therapy, or Antiviral Therapy, or Both for COVID-19: A Systematic Review. Drugs 2020; 80(18): 1929-46.

42. Atal S, Fatima Z. IL-6 Inhibitors in the Treatment of Serious COVID-19: A Promising Therapy? Pharmaceut Med 2020; 34(4): 223-31.

43. Capra R, De Rossi N, Mattioli F, et al. Impact of low dose tocilizumab on mortality rate in patients with COVID-19 related pneumonia. Eur J Intern Med 2020; 76: 31-5.

44. Colaneri M, Bogliolo L, Valsecchi P, et al. Tocilizumab for Treatment of Severe COVID-19 Patients: Preliminary Results from SMAtteo COvid19 REgistry (SMACORE). Microorganisms 2020; 8(5).

45. Guaraldi G, Meschiari M, Cozzi-Lepri A, et al. Tocilizumab in patients with severe COVID-19: a retrospective cohort study. Lancet Rheumatol 2020; 2(8): e474-e84.

46. Klopfenstein T, Zayet $S$, Lohse A, et al. Tocilizumab therapy reduced intensive care unit admissions and/or mortality in COVID-19 patients. Med Mal Infect 2020; 50(5): 397-400.

47. Toniati P, Piva S, Cattalini M, et al. Tocilizumab for the treatment of severe COVID-19 pneumonia with hyperinflammatory syndrome and acute respiratory failure: A single center study of 100 patients in Brescia, Italy. Autoimmunity reviews 2020; 19(7): 102568.

48. Xu X, Han M, Li T, et al. Effective treatment of severe COVID-19 patients with tocilizumab. Proc Natl Acad Sci U S A 2020; 117(20): 10970-5.

49. Budhathoki P, Shrestha DB, Rawal E, etal. Corticosteroids in COVID-19: Is it Rational? A Systematic Review and Meta-Analysis. SN Compr Clin Med 2020: 1-21.

50. Mishra GP, Mulani J. Corticosteroids for COVID-19: the search for an optimum duration of therapy. Lancet Respir Med 2021; 9(1): e8. 
51. Bracken MB. Steroids for acute spinal cord injury. Cochrane Database Syst Rev 2012; 1: CD001046.

52. Hurlbert RJ. Methylprednisolone for acute spinal cord injury: an inappropriate standard of care.J Neurosurg 2000; 93(1 Suppl): 1-7.

53. Alderson P, Roberts I. Corticosteroids for acute traumatic brain injury. Cochrane Database Syst Rev 2005; (1): CD000196.

54. Cofano F, Boido M, Monticelli M, et al. Mesenchymal Stem Cells for Spinal Cord Injury: Current Options, Limitations, and Future of Cell Therapy. International journal of molecular sciences 2019; 20(11).

55. Jin MC, Medress ZA, Azad TD, et al. Stem cell therapies for acute spinal cord injury in humans: a review. Neurosurg Focus 2019; 46(3): E10.
56. Edwards CJ. Immunological therapies for rheumatoid arthritis. $\mathrm{Br}$ Med Bull 2005; 73-74: 71-82.

57. Isaacs JD, Ferraccioli G. The need for personalised medicine for rheumatoid arthritis. Ann Rheum Dis 2011; 70(1): 4-7.

58. Verweij CL. Predicting the future of anti-tumor necrosis factor therapy. Arthritis Res Ther 2009; 11(3): 115.

59. De Bari C. Are mesenchymal stem cells in rheumatoid arthritis the good or bad guys? Arthritis Res Ther 2015; 17: 113.

60. Caplan AI. Mesenchymal Stem Cells: Time to Change the Name! Stem cells translational medicine $2017 ; 6(6): 1445-51$. 